\title{
ON FREDHOLM TYPE INTEGRODIFFERENTIAL EQUATION
}

\author{
B. G. PACHPATTE
}

\begin{abstract}
In this paper we study the existence, uniqueness and other properties of solutions of a Fredholm type integrodifferential equation. The main tools employed in the analysis are based on the applications of the Banach fixed point theorem and a certain integral inequality with explicit estimate.
\end{abstract}

\section{Introduction}

Consider the nonlinear Fredholm type integrodifferential equation

$$
x(t)=g(t)+\int_{a}^{b} f\left(t, s, x(s), x^{\prime}(s), \ldots, x^{(n-1)}(s)\right) d s
$$

for $a \leq t \leq b$, where $x, g, f$ are real valued functions and $n \geq 2$ is an integer. Let $R$ denote the set of real numbers, $E=R \times \cdots \times R$ ( $n$ times) be the product space and $I=[a, b], R_{+}=[0, \infty$ ) be the given subsets of $R$. Let $C(A, B)$ denotes the class of continuous functions from the set $A$ to the set $B$. We assume that $g \in C(I, R), f \in C\left(I^{2} \times E, R\right)$ and are continuously $(n-1)$-times differentiable with respect to $t$, on the respective domains of their definitions.

Many papers have been devoted to the study of special versions of equation (1.1) when $n=$ 1 by using different techniques, see $[5,6,7]$ and the references given therein. In a recent paper [1] , the authors have studied the existence, uniqueness and approximation of solutions of equation (1.1) in Banach spaces when $n=2$ by using Perov's fixed point theorem, the method of successive approximations and trapezoidal quadrature rule. The main objective of this paper is to study the existence, uniqueness and other properties of the solutions of equation (1.1) under various assumptions on the functions involved therein. The well known Banach fixed point theorem ( see [5, p.37]) coupled with Bielecki type norm (see [2]) and the integral inequality with explicit estimate established by Pachpatte (see [9, p.41]) are used to establish the results.

Received March 30, 2007; revised September 10, 2007.

2000 Mathematics Subject Classification. 34K10, 35R10.

Key words and phrases. Fredholm type, integrodifferential equation, Banach fixed point theorem, integral inequality, Bielecki type norm, existence and uniqueness, estimates on solutions, continuous dependence. 


\section{Existence and uniqueness}

For continuous functions $u^{(j)}(t): I \rightarrow R(j=0,1, \ldots, n-1)$, we denote by $|u(t)|_{E}=\sum_{j=0}^{n-1}\left|u^{(j)}(t)\right|$. Let $S$ be a space of those continuous functions $u(t): I \rightarrow R$ which are $(n-1)$-times continuously differentiable, $\left(u(t), u^{\prime}(t), \ldots, u^{(n-1)}(t)\right) \in E$ and fulfil the condition

$$
|u(t)|_{E}=o(\exp (\lambda t))
$$

for $t \in I$, where $\lambda$ is a positive constant. In the space $S$ we define the norm (see $[2,4,8,10])$

$$
|u|_{S}=\sup _{t \in I}\left\{|u(t)|_{E} \exp (-\lambda t)\right\}
$$

It is easy to see that $S$ with norm defined in (2.2) is a Banach space. We note that the condition (2.1) implies that there exists a nonnegative constant $N$ such that

$$
|u(t)|_{E} \leq N \exp (\lambda t) .
$$

Using this fact in (2.2) we observe that

$$
|u|_{S} \leq N
$$

By a solution of equation (1.1) we mean a continuous function $x(t), t \in I$ which is continuously $(n-1)$-times differentiable with respect to $t$ and satisfies the equation (1.1). It is easy to observe that the solution $x(t)$ of equation (1.1) and its derivatives satisfy the integral equations (see [3, p. 318])

$$
x^{(j)}(t)=g^{(j)}(t)+\int_{a}^{b} \frac{\partial^{j}}{\partial t^{j}} f\left(t, s, x(s), x^{\prime}(s), \ldots, x^{(n-1)}(s)\right) d s,
$$

for $j=0,1, \ldots, n-1$.

We need the following special version of the integral inequality established by Pachpatte (see [9, p.41]). We shall state it in the following lemma for completeness.

Lemma. Let $u(t), p(t), q(t), r(t) \in C\left(I, R_{+}\right)$and suppose that

$$
u(t) \leq p(t)+q(t) \int_{a}^{b} r(s) u(s) d s
$$

for $t \in I$. If

$$
d=\int_{a}^{b} r(s) q(s) d s<1
$$

then

for $t \in I$.

$$
u(t) \leq p(t)+q(t)\left\{\frac{1}{1-d} \int_{a}^{b} r(s) p(s) d s\right\}
$$

The following theorem concerning the existence of a unique solution of equation (1.1) holds.

Theorem 1. Assume that 
(i) the function $f$ in equation (1.1) and its derivatives with respect to $t$ satisfy the conditions

$$
\begin{aligned}
& \left|\frac{\partial^{j}}{\partial t^{j}} f\left(t, s, u_{0}, u_{1}, \ldots, u_{n-1}\right)-\frac{\partial^{j}}{\partial t^{j}} f\left(t, s, v_{0}, v_{1}, \ldots, v_{n-1}\right)\right| \\
& \quad \leq r_{j}(t, s) \sum_{i=0}^{n-1}\left|u_{i}-v_{i}\right|
\end{aligned}
$$

for $j=0,1, \ldots, n-1$, where $r_{j}(t, s) \in C\left(I^{2}, R_{+}\right)$,

(ii) for $j=0,1, \ldots, n-1$ there exist nonnegative constants $\alpha_{j}$ such that $\sum_{j=0}^{n-1} \alpha_{j}<1$ and

$$
\int_{a}^{b} r_{j}(t, s) \exp (\lambda s) d s \leq \alpha_{j} \exp (\lambda t)
$$

for $t \in I$, where $\lambda$ is as given in (2.1),

(iii) for $j=0,1, \ldots, n-1$ there exist nonnegative constants $P_{j}$ such that

$$
\left|g^{(j)}(t)\right|+\int_{a}^{b}\left|\frac{\partial^{j}}{\partial t^{j}} f(t, s, 0,0, \ldots, 0)\right| d s \leq P_{j} \exp (\lambda t)
$$

where $g, f$ are as given in equation (1.1) and $\lambda$ is as given in (2.1).

Then equation (1.1) has a unique solution $x(t)$ in $S$ on $I$.

Proof. Let $x(t) \in S$ and define the operator

$$
(T x)(t)=g(t)+\int_{a}^{b} f\left(t, s, x(s), x^{\prime}(s), \ldots, x^{(n-1)}(s)\right) d s .
$$

Differentiating both sides of (2.8) with respect to $t$ (see [3, p.318]) we have

$$
(T x)^{(j)}(t)=g^{(j)}(t)+\int_{a}^{b} \frac{\partial^{j}}{\partial t^{j}} f\left(t, s, x(s), x^{\prime}(s), \ldots, x^{(n-1)}(s)\right) d s,
$$

for $j=0,1, \ldots, n-1$. Now, we show that $(T x)^{(j)}$ for $j=0,1, \ldots, n-1$, maps $S$ into itself. Evidently, $(T x)^{(j)}$ are continuous on $I$ and $(T x)^{(j)} \in R$. We verify that (2.1) is fulfilled. From (2.8), 
(2.9), using the hypotheses and (2.3) we have

$$
\begin{aligned}
|(T x)(t)|_{E}= & \sum_{j=0}^{n-1}\left|(T x)^{(j)}(t)\right| \\
\leq & \sum_{j=0}^{n-1}\left|g^{(j)}(t)\right|+\sum_{j=0}^{n-1} \int_{a}^{b} \mid \frac{\partial^{j}}{\partial t^{j}} f\left(t, s, x(s), x^{\prime}(s), \ldots, x^{(n-1)}(s)\right) \\
& -\frac{\partial^{j}}{\partial t^{j}} f(t, s, 0,0, \ldots, 0)\left|d s+\sum_{j=0}^{n-1} \int_{a}^{b}\right| \frac{\partial^{j}}{\partial t^{j}} f(t, s, 0,0, \ldots, 0) \mid d s \\
\leq & \sum_{j=0}^{n-1} P_{j} \exp (\lambda t)+\sum_{j=0}^{n-1} \int_{a}^{b} r_{j}(t, s)|x(s)|_{E} d s \\
\leq & \sum_{j=0}^{n-1} P_{j} \exp (\lambda t)+|x|_{S} \sum_{j=0}^{n-1} \int_{a}^{b} r_{j}(t, s) \exp (\lambda s) d s \\
\leq & {\left[\sum_{j=0}^{n-1} P_{j}+N \sum_{j=0}^{n-1} \alpha_{j}\right] \exp (\lambda t) . }
\end{aligned}
$$

From (2.10) it follows that $(T x) \in S$. This proves that $T$ maps $S$ into itself.

Now, we verify that the operator $T$ is a contraction map. Let $x(t), y(t) \in S$. From (2.8), (2.9) and using the hypotheses we have

$$
\begin{aligned}
|(T x)(t)-(T y)(t)|_{E}= & \sum_{j=0}^{n-1}\left|(T x)^{(j)}(t)-(T y)^{(j)}(t)\right| \\
\leq & \sum_{j=0}^{n-1} \int_{a}^{b} \mid \frac{\partial^{j}}{\partial t^{j}} f\left(t, s, x(s), x^{\prime}(s), \ldots, x^{(n-1)}(s)\right) \\
& -\frac{\partial^{j}}{\partial t^{j}} f\left(t, s, y(s), y^{\prime}(s), \ldots, y^{(n-1)}(s)\right) \mid d s \\
\leq & \sum_{j=0}^{n-1} \int_{a}^{b} r_{j}(t, s)|x(s)-y(s)|_{E} d s \\
\leq & |x-y|_{S} \sum_{j=0}^{n-1} \int_{a}^{b} r_{j}(t, s) \exp (\lambda s) d s \\
\leq & |x-y|_{s} \sum_{j=0}^{n-1} \alpha_{j} \exp (\lambda t) .
\end{aligned}
$$

From (2.11) we obtain

$$
|T x-T y|_{S} \leq \sum_{j=0}^{n-1} \alpha_{j}|x-y|_{S} .
$$

Since $\sum_{j=0}^{n-1} \alpha_{j}<1$, it follows from Banach fixed point theorem (see [5, p.37]) that $T$ has a unique fixed point in $S$. The fixed point of $T$ is however a solution of equation (1.1). The proof is complete. 
Remark 1. We note that in 1956 A. Bielecki [2] first used the norm defined in (2.2) for proving global existence and uniqueness of solutions of ordinary differential equations. For developments related to this topic, see [4].

Indeed, the following theorem is true concerning the uniqueness of solutions of equation (1.1) without existence part.

Theorem 2. Assume that the function $f$ in equation (1.1) and its derivatives satisfy the conditions (2.5) with $r_{j}(t, s)=k(t) e_{j}(s)$ for $j=0,1, \ldots, n-1$, where $k, e_{j} \in C\left(I, R_{+}\right)$. Let $e(t)=$ $\sum_{j=0}^{n-1} e_{j}(t)$ and suppose that

$$
d_{1}=\int_{a}^{b} e(s) k(s) d s<1 .
$$

Then the equation (1.1) has at most one solution on I.

Proof. Let $x(t)$ and $y(t)$ be two solutions of equation (1.1). Then from (2.4) we have

$$
\begin{aligned}
\sum_{j=0}^{n-1}\left|x^{(j)}(t)-y^{(j)}(t)\right| \leq & \sum_{j=0}^{n-1} \int_{a}^{b} \mid \frac{\partial^{j}}{\partial t^{j}} f\left(t, s, x(s), x^{\prime}(s), \ldots, x^{(n-1)}(s)\right) \\
& -\frac{\partial^{j}}{\partial t^{j}} f\left(t, s, y(s), y^{\prime}(s), \ldots, y^{(n-1)}(s)\right) \mid d s \\
\leq & k(t) \int_{a}^{b} e(s) \sum_{j=0}^{n-1}\left|x^{(j)}(s)-y^{(j)}(s)\right| d s .
\end{aligned}
$$

Now, an application of Lemma (when $p(t)=0$ ) to (2.13) yields

$$
\sum_{j=0}^{n-1}\left|x^{(j)}(t)-y^{(j)}(t)\right| \leq 0
$$

and hence $x(t)=y(t)$, which proves the uniqueness of solutions of equation (1.1) on $I$.

\section{Estimates on the solutions}

In this section we obtain estimates on the solutions of equation (1.1) under some suitable conditions on the functions involved therein.

The following theorem concerning the estimate on the solution of equation (1.1) holds.

Theorem 3. Assume that the functions $g, f$ in equation (1.1) and their derivatives with respect to $t$ satisfy the conditions

$$
\begin{gathered}
\sum_{j=0}^{n-1}\left|g^{(j)}(t)\right| \leq h(t), \\
\left|\frac{\partial^{j}}{\partial t^{j}} f\left(t, s, u_{0}, u_{1}, \ldots, u_{n-1}\right)\right| \leq c(t) e_{j}(s) \sum_{i=0}^{n-1}\left|u_{i}\right|,
\end{gathered}
$$


for $j=0,1, \ldots, n-1$, where $h, c, e_{j} \in C\left(I, R_{+}\right)$. Let $e(t)=\sum_{j=0}^{n-1} e_{j}(t)$ and suppose that

$$
d_{2}=\int_{a}^{b} e(s) c(s) d s<1 .
$$

If $x(t), t \in I$ is any solution of equation (1.1), then

$$
\sum_{j=0}^{n-1}\left|x^{(j)}(t)\right| \leq h(t)+c(t)\left\{\frac{1}{1-d_{2}} \int_{a}^{b} e(s) h(s) d s\right\}
$$

for $t \in I$.

Proof. Using the fact that $x(t), t \in I$. is a solution of equation (1.1) and hypotheses we have

$$
\begin{aligned}
\sum_{j=0}^{n-1}\left|x^{(j)}(t)\right| & \leq \sum_{j=0}^{n-1}\left|g^{(j)}(t)\right|+\sum_{j=0}^{n-1} \int_{a}^{b}\left|\frac{\partial^{j}}{\partial t^{j}} f\left(t, s, x(s), x^{\prime}(s), \ldots, x^{(n-1)}(s)\right)\right| d s \\
& \leq h(t)+\sum_{j=0}^{n-1} \int_{a}^{b} c(t) e_{j}(s) \sum_{i=0}^{n-1}\left|x^{(i)}(s)\right| d s \\
& =h(t)+c(t) \int_{a}^{b} e(s) \sum_{j=0}^{n-1}\left|x^{(j)}(s)\right| d s .
\end{aligned}
$$

Now an application of Lemma to (3.5) yields (3.4).

Remark 2. It is interesting to note that the estimate obtained in (3.4) yields not only the bound on the solution of equation (1.1) but also the bound on their derivatives. It is obvious that the solution $x(t)$ of equation (1.1) and its derivatives $x^{(j)}(t), j=1,2, \ldots, n-1$, are bounded, since they are continuous on the closed and finite interval $I$.

Next, we shall obtain the estimate on the solution of equation (1.1) assuming that the function $f$ and its derivatives with respect to $t$ satisfy the Lipschitz type conditions.

Theorem 4. Assume that the hypotheses of Theorem 2 hold and let

$$
\alpha(t)=\sum_{j=0}^{n-1} \int_{a}^{b}\left|\frac{\partial^{j}}{\partial t^{j}} f\left(t, s, g(s), g^{\prime}(s), \ldots, g^{(n-1)}(s)\right)\right| d s,
$$

for $t \in I$. If $x(t), t \in I$, is any solution of equation (1.1), then

$$
\sum_{j=0}^{n-1}\left|x^{(j)}(t)-g^{(j)}(t)\right| \leq \alpha(t)+k(t)\left\{\frac{1}{1-d_{1}} \int_{a}^{b} e(s) \alpha(s) d s\right\},
$$

for $t \in I$. 
Proof. Since $x(t)$ is a solution of equation (1.1), from (2.4) and using the hypotheses we have

$$
\begin{aligned}
\sum_{j=0}^{n-1}\left|x^{(j)}(t)-g^{(j)}(t)\right| \leq & \sum_{j=0}^{n-1} \int_{a}^{b} \mid \frac{\partial^{j}}{\partial t^{j}} f\left(t, s, x(s), x^{\prime}(s), \ldots, x^{(n-1)}(s)\right) \\
& -\frac{\partial^{j}}{\partial t^{j}} f\left(t, s, g(s), g^{\prime}(s), \ldots, g^{(n-1)}(s)\right) \mid d s \\
& +\sum_{j=0}^{n-1} \int_{a}^{b}\left|\frac{\partial^{j}}{\partial t^{j}} f\left(t, s, g(s), g^{\prime}(s), \ldots, g^{(n-1)}(s)\right)\right| d s \\
\leq & \alpha(t)+k(t) \int_{a}^{b} e(s) \sum_{j=0}^{n-1}\left|x^{(j)}(s)-g^{(j)}(s)\right| d s .
\end{aligned}
$$

Now an application of Lemma to (3.8) yields (3.7).

\section{Continuous dependence}

Consider the equation (1.1) and the following Fredholm integrodifferential equation

$$
y(t)=G(t)+\int_{a}^{b} F\left(t, s, y(s), y^{\prime}(s), \ldots, y^{(n-1)}(s)\right) d s,
$$

for $t \in I$ and $n \geq 2$ is an integer, where $y, G, F$ are real valued functions. We assume that $G \in$ $C(I, R), F \in C\left(I^{2}, R\right)$ and are continuously $(n-1)$-times differentiable with respect to $t$.

The following theorem deals with the continuous dependence of solution of equation (1.1) on the functions involved therein.

Theorem 5. Assume that the hypotheses of Theorem 2 hold. Suppose that

$$
\begin{gathered}
\sum_{j=0}^{n-1}\left|g^{(j)}(t)-G^{(j)}(t)\right|+\sum_{j=0}^{n-1} \mid \frac{\partial^{j}}{\partial t^{j}} f\left(t, s, y(s), y^{\prime}(s), \ldots, y^{(n-1)}(s)\right) \\
-\frac{\partial^{j}}{\partial t^{j}} f\left(t, s, y(s), y^{\prime}(s), \ldots, y^{(n-1)}(s)\right) \mid d s \leq \beta(t),
\end{gathered}
$$

where $g, f$ and $G, F$ are the functions involved in equations (1.1) and (4.1), $\beta \in C\left(I, R_{+}\right)$and $y(t)$ is a solution of equation (4.1) for $t \in I$. Then the solution $x(t), t \in I$ of equation (1.1) depends continuously on the functions involved on the right hand side of equation (1.1).

Proof. Using the facts that $x(t)$ and $y(t)$ are the solutions of equations (1.1) and (4.1) and 
the hypotheses we have

$$
\begin{aligned}
\sum_{j=0}^{n-1}\left|x^{(j)}(t)-y^{(j)}(t)\right| \leq & \sum_{j=0}^{n-1}\left|g^{(j)}(t)-G^{(j)}(t)\right| \\
& +\sum_{j=0}^{n-1} \int_{a}^{b} \mid \frac{\partial^{j}}{\partial t^{j}} f\left(t, s, x(s), x^{\prime}(s), \ldots, x^{(n-1)}(s)\right) \\
& -\frac{\partial^{j}}{\partial t^{j}} f\left(t, s, y(s), y^{\prime}(s), \ldots, y^{(n-1)}(s)\right) \mid d s \\
& +\sum_{j=0}^{n-1} \int_{a}^{b} \mid \frac{\partial^{j}}{\partial t^{j}} f\left(t, s, y(s), y^{\prime}(s), \ldots, y^{(n-1)}(s)\right) \\
& -\frac{\partial^{j}}{\partial t^{j}} F\left(t, s, y(s), y^{\prime}(s), \ldots, y^{(n-1)}(s)\right) \mid d s \\
\leq & \beta(t)+k(t) \int_{a}^{b} e(s) \sum_{j=0}^{n-1}\left|x^{(j)}(s)-y^{(j)}(s)\right| d s .
\end{aligned}
$$

Now an application of Lemma to (4.3) yields

$$
\sum_{j=0}^{n-1}\left|x^{(j)}(t)-y^{(j)}(t)\right| \leq \beta(t)+k(t)\left\{\frac{1}{1-d_{1}} \int_{a}^{b} e(s) \beta(s) d s\right\} .
$$

From (4.4) it follows that the solutions of equation (1.1) depend continuously on the functions involved on the right hand side of equation (1.1).

Next, we consider the following Fredholm type integrodifferential equations

$$
z(t)=g(t)+\int_{a}^{b} f\left(t, s, z(s), z^{\prime}(s), \ldots, z^{(n-1)}(s), \mu\right) d s
$$

and

$$
z(t)=g(t)+\int_{a}^{b} f\left(t, s, z(s), z^{\prime}(s), \ldots, z^{(n-1)}(s), \mu_{0}\right) d s
$$

for $t \in I$ and $n \geq 2$ is an integer, where $z, g, f$ are real valued functions and $\mu, \mu_{0}$ are real parameters. We assume that $g \in C(I, R), f \in C\left(I^{2} \times E \times R, R\right)$ and are continuously $(n-1)$-times differentiable with respect to $t$.

Finally, we present the following theorem which deals with the dependency of solutions of equations (4.5) and (4.6) on parameters.

Theorem 6. Assume that the function $f$ in equations (4.5) and (4.6) and its derivatives with 
respect to $t$ satisfy the conditions

$$
\begin{aligned}
& \left|\frac{\partial^{j}}{\partial t^{j}} f\left(t, s, u_{0}, u_{1}, \ldots, u_{n-1}, \mu\right)-\frac{\partial^{j}}{\partial t^{j}} f\left(t, s, \bar{u}_{0}, \bar{u}_{1}, \ldots, \bar{u}_{n-1}, \mu\right)\right| \\
& \quad \leq \bar{q}(t) \bar{e}_{j}(s) \sum_{i=0}^{n-1}\left|u_{i}-\bar{u}_{i}\right|, \\
& \quad\left|\frac{\partial^{j}}{\partial t^{j}} f\left(t, s, u_{0}, u_{1}, \ldots, u_{n-1}, \mu\right)-\frac{\partial^{j}}{\partial t^{j}} f\left(t, s, u_{0}, u_{1}, \ldots, u_{n-1}, \mu_{0}\right)\right| \\
& \quad \leq \gamma_{j}(t, s)\left|\mu-\mu_{0}\right|,
\end{aligned}
$$

for $j=0,1, \ldots, n-1$, where $\bar{q}, \bar{e}_{j} \in C\left(I, R_{+}\right), \gamma_{j} \in C\left(I^{2}, R_{+}\right)$. Let $\bar{e}(t)=\sum_{j=0}^{n-1} \bar{e}_{j}(t), \gamma(t)=\int_{a}^{b} \gamma_{j}(t, s) d s$ and suppose that

$$
\bar{d}_{2}=\int_{a}^{b} \bar{e}(s) \bar{q}(s) d s<1 .
$$

Let $z_{1}(t)$ and $z_{2}(t)$ are the solutions of equations (4.5) and (4.6) respectively. Then

$$
\sum_{j=0}^{n-1}\left|z_{1}^{(j)}(t)-z_{2}^{(j)}(t)\right| \leq\left|\mu-\mu_{0}\right|\left[\gamma(t)+\bar{q}(t)\left\{\frac{1}{1-\bar{d}_{2}} \int_{a}^{b} \bar{e}(s) \gamma(s) d s\right\}\right],
$$

for $t \in I$.

Proof. Using the facts that $z_{1}(t)$ and $z_{2}(t)$ are the solutions of equations (4.5) and (4.6) and the hypotheses we have

$$
\begin{aligned}
\sum_{j=0}^{n-1}\left|z_{1}^{(j)}(t)-z_{2}^{(j)}(t)\right| \leq & \sum_{j=0}^{n-1} \int_{a}^{b} \mid \frac{\partial^{j}}{\partial t^{j}} f\left(t, s, z_{1}(s), z_{1}^{\prime}(s), \ldots, z_{1}^{(n-1)}(s), \mu\right) \\
& -\frac{\partial^{j}}{\partial t^{j}} f\left(t, s, z_{2}(s), z_{2}^{\prime}(s), \ldots, z_{2}^{(n-1)}(s), \mu\right) \mid d s \\
& +\sum_{j=0}^{n-1} \int_{a}^{b} \mid \frac{\partial^{j}}{\partial t^{j}} f\left(t, s, z_{2}(s), z_{2}^{\prime}(s), \ldots, z_{2}^{(n-1)}(s), \mu\right) \\
& -\frac{\partial^{j}}{\partial t^{j}} f\left(t, s, z_{2}(s), z_{2}^{\prime}(s), \ldots, z_{2}^{(n-1)}(s), \mu_{0}\right) \mid d s \\
\leq & \bar{q}(t) \int_{a}^{b} \sum_{j=0}^{n-1} \bar{e}_{j}(s) \sum_{i=0}^{n-1}\left|z_{1}^{(i)}(s)-z_{2}^{(i)}(s)\right| d s \\
& +\left|\mu-\mu_{0}\right| \int_{a}^{b} \sum_{j=0}^{n-1} \gamma_{j}(t, s) d s \\
= & \left|\mu-\mu_{0}\right| \gamma(t)+\bar{q}(t) \int_{a}^{b} \bar{e}(s) \sum_{j=0}^{n-1}\left|z_{1}^{(j)}(s)-z_{2}^{(j)}(s)\right| d s .
\end{aligned}
$$

Now an application of Lemma to (4.11) yields (4.10), which shows the dependency of solutions of equations (4.5) and (4.6) on parameters. 
Remark 3. We note that our approach to the study of equation (1.1) is different from the one used in [1] and we believe that the results given here are of independent interest.

\section{References}

[1] A. M. Bica, V. A. Căuş and S. Mureşan, Application of a trapezoid tnequality to neutral Fredholm integro-differential equations in Banach spaces, J. Inequal Pure and Appl. Math. 7(2006), Art.173.

[2] A. Bielecki, Une remarque sur la méthod de Banach-Cacciopoli-Tikhonov dans la théorie des équations différentilles ordinaries, Bull. Acad. Polon. Sci. Sér. Sci. Math. Phys. Astr. 4(1956), 261-264.

[3] B. Cahlon and D. Westreich, Complete continuity of integro-differential operators with discontinuous kernels and collectively compact approximations, J. Math. Anal. Appl. 71(1979), 313-332.

[4] C. Corduneanu, Bielecki's method in the theory of integral equations, Ann. Univ. Mariae CurieSklodawskia, Section A, 38(1984), 23-40.

[5] C. Corduneanu, Integral Equations and Applications, Cambridge University Press, 1991.

[6] M. A. Krasnoselskii, Topological Methods in the Theory of Nonlinear Integral Equations, Pergamon Press, Oxford, 1964.

[7] R.K. Miller, Nonlinear Volterra Integral Equations, W.A.Benjamin, Menlo Park CA, 1971.

[8] B. G. Pachpatte, On a nonlinear Volterra Integral-Functional equation, Funkcialaj Ekvacioj 26(1983), 1-9.

[9] B. G. Pachpatte, Integral and Finite Difference Inequalities and Applications, North-Holland Mathematics Studies 205, Elsevier Science B. V., 2006.

[10] B. G. Pachpatte, On higher order Volterra-Fredholm integrodifferential equation, Fasciculi Mathematici Nr. 37(2007), 35-48.

57 Shri Niketan Colony, Near Abhinay Talkies, Aurangabad 431001 (Maharashtra) India.

E-mail: bgpachpatte@gmail.com 